COMMENTARY. Diazepam is FDA approved for the treatment of pediatric status epilepticus but lorazepam is not approved. Several reports of treatment with lorazepam have suggested superior effectiveness, longer duration of action, and lower incidence of respiratory depression when compared to diazepam, but the evidence to support lorazepam superiority is inconclusive. In one study, intravenously administered lorazepam was compared to rectal diazepam [1]. In a Cochrane Database Review [2], intravenous lorazepam is as effective as intravenous diazepam, has fewer adverse events, and rectal lorazepam may be more effective than rectal diazepam. Where intravenous access is unavailable, buccal midazolam is recommended as the treatment of choice and intranasal lorazepam is as effective as intravenous diazepam. In contrast to these positive lorazepam responses, the results of the present study do not support the preferred use of lorazepam vs diazepam in the treatment of pediatric convulsive status epilepticus [3]. Neither agent is optimal since SE is uncontrolled in 1 in 4 children and severe respiratory depression occurs in approximately 1 in 6.

The FEBSTAT study of the emergency management of febrile status epilepticus finds that the earlier the onset of treatment, the shorter the total seizure duration and better the outcome [4]. A comparison of buccal or intranasal midazolam vs intravenous or rectal diazepam found that non-IV midazolam was as effective as IV diazepam, and buccal midazolam was superior to rectal diazepam in achieving seizure control; and respiratory complications requiring intervention were similar, regardless of administration route [5]. A comparison of midazolam nasal spray and rectal diazepam solution for residential treatment of seizure exacerbations found midazolam was equal in efficacy to diazepam, and drowsiness occurred in more than $50 \%$ of administrations for both drugs. The majority of patients and caregivers preferred the nasal spray to rectal formulation [6]. In the UK, an epidemiological study strongly supports prehospital treatment with buccal midazolam as a widely used but unlicensed option in the community [1].

\footnotetext{
References.

1. Neville BG, et al. Acta Neurol Scand. 2007 Apr;115(4 Suppl):21-4.

2. Appleton R, et al. Cochrane Database Syst Rev. 2008 Jul 16;(3):CD001905.

3. Chamberlain JM, et al. JAMA. 2014 Apr 23-30;311(16):1652-60.

4. Seinfeld S, et al. Epilepsia. 2014 Mar;55(3):388-95.

5. McMullan J, et al. Acad Emerg Med. 2010 Jun;17(6):575-82.

6. De Haan GJ, et al. Epilepsia. 2010 Mar;51(3):478-82.
}

\title{
PYRIDOXINE RESPONSIVENESS AND PNPO GENE MUTATIONS
}

Investigators at University Hospital, Zurich, Switzerland, and multiple centers in Europe and Canada, sequenced the pyridoxal 5-phosphate oxidase (PNPO) gene in 31 patients with pyridoxine-responsive seizures but normal biomarkers for antiquitin deficiency and normal sequencing of the ALDH7A1 gene. Eleven patients from 7 families carried 3 novel mutations of the PNPO gene. Response to pyridoxine was prompt in 4 patients, delayed in 2, on EEG only in 2, and initially absent in another 2 patients. Earlier and continuous pyridoxine therapy was related to a better prognosis. Two unrelated patients homozygous for the pArg225His mutation developed status epilepticus when switched to pyridoxal 5-phosphate (PLP). 
The findings shift the paradigm of exclusive PLP responsiveness of patients with PNPO deficiency and contradict the strategy of using PLP instead of pyridoxine as the first-line vitamin to test for all inborn errors with vitamin B6-responsive seizures. Testing for PNPO mutations is important in pyridoxine-responsive patients with normal biomarkers for antiquitin deficiency or other B6-dependent neonatal epilepsies. A sequential trial with pyridoxine and PLP should be performed routinely in neonates with AED-resistant seizures, irrespective of a history of birth asphyxia. The challenge of recognizing a delayed pyridoxine effect and lack of specific biomarkers caries a risk of misdiagnosis. (Plecko B, Paul K, Mills P, et al. Pyridoxine responsiveness in novel mutations of the PNPO gene. Neurology 2014 Apr 22;82(16):1425-33).

COMMENTARY. In an editorial [1], the clinical characteristics of pyridoxine dependent epilepsy (PDE) and PLP-dependent cases are differentiated by their presentation with full-term and premature birth, respectively. The EEG of PDE has a classic burst-suppression pattern, whereas the PNPO deficiency is associated with a nonspecific and less paroxysmal pattern. Cases of partial pyridoxine responsiveness have PNPO mutations.

Congenital brain malformations and pyridoxine dependent epilepsy. Several cases of brain malformation are reported in association with pyridoxine dependent epilepsy (PDE), and a current case-report concerns an infant with seizures at 7 days, initially responsive to phenobarbital, later diagnosed with PDE caused by ALDH7A1 genetic defect. Brain sonography on day 1 and MRI on day 5 confirmed bilateral asymmetric ventriculomegaly caused by bilateral subependymal cysts. At age 7 months he was seizure free on pyridoxine $200 \mathrm{mg} /$ day $(29 \mathrm{mg} / \mathrm{kg}$ per day), neurological examination was unremarkable, EEG was normal, and ventriculomegaly was resolved. Despite the structural brain malformation, PDE should always be considered in the differential diagnosis of neonatal seizures refractory to treatment with AEDs [2].

\section{References.}

1. Pearl PL, Gospe SM Jr. Neurology. 2014 Apr 22;82(16):1392-4.

2. Jain-Ghai S, et al. Pediatrics. 2014 Apr;133(4):e1092-6.

\section{ETHOSUXIMIDE VS VALPROATE LONG-TERM REMISSION OF ABSENCE EPILEPSY}

Investigators from the Epilepsy Center, Lurie Children's Hospital of Chicago, and Yale School of Medicine, New Haven, CT, examined the possible association between long-term seizure outcome of childhood absence epilepsy (CAE) and the initial treatment with ethosuximide (ESM) or valproic acid (VPA). Newly diagnosed patients throughout the State of Connecticut from 1993 to 1997 were identified through the offices of 16 of 17 child neurologists and data were also reviewed by three pediatric epileptologists. Initial success rates were $59 \%$ of 41 treated with ESM and $56 \%$ of 18 who received VPA. Early remission and drug resistance were similar in each group. Complete remission (seizure free for 5 years and 5 years off medication) occurred in $31(76 \%)$ treated with ESM and $7(39 \%)$ who received VPA ( $p=0.007)$. ESM was associated with a higher rate of complete remission than VPA. In 53 children followed $>10$ years, remission was $76 \%$ 